\title{
Detection of Infected Leaves and Botanical Diseases using Curvelet Transform
}

\author{
Nazish Tunio*1, Abdul Latif Memon*2, Faheem Yar Khuhawar*3 and Ghulam Mustafa Abro ${ }^{\dagger 4}$ \\ *Dept. of Telecommunication Engineering, MUET, Jamshoro, PK \\ ${ }^{\dagger}$ Dept. of Electronic Eng., Hamdard University, Karachi
}

\begin{abstract}
The study of plants is known as botany and for any botanist it is a daily routine work to examine various plants in their research lab. This research efforts an image processingbased algorithm for extracting the region of interest (ROI) from plant leaf in order to classify the specie and to recognize the particular botanical disease as well. Moreover, this paper addresses the implementation of curvelet transform on subdivided leaf images in order to compute the related information and train the support vector machine (SVM) classifier to execute better results. Furthermore, the paper presents a comparative analysis of existing and proposed algorithm for species and botanical diseases recognition over the dataset of leaves. The proposed multi-dimensional curvelet transform based algorithm provides relatively greater accuracy of $93.5 \%$ with leaves dataset.
\end{abstract}

Keywords-Region Of Interest (ROI); Support Vector Machine (SVM); feature extraction; curvelet transform; alternata; anthracnose; blightness

\section{INTRODUCTION}

An image that we see with our eyes is analogous in nature to the image captured via digital camera. By definition, an image is nothing but a matrix of different intensity levels and treated as a two-dimensional signal in MATLAB software. One may perform the digital image processing due to two major reasons:

- To enhance the pictorial information of any distorted or an old image.

- To render the image so that any of the computing gadget may understand it.

Our work focuses on the second postulate mainly and for this purpose, one should acquire methods related to recognition techniques. It is one of the imperative method in several applications since last few years, such as face detection, medical imaging and agricultural applications. This paper demonstrates the identification of plant species along with the analysis in identifying the botanical diseases available in leaf images. A normal human eye can not tackle such complicated recognition based tasks and thus it will be requiring a support that will assist us in identifying such types of problems. That support comes from image processing-oriented algorithms.

In today's world, modern agronomy, breeding of plants and pesticides have increased the production of agricultural goods. However, usage of excessive pesticides has engendered an ecological damage too. Hence, in such conditions, a computer vision-based algorithm can reduce this damage by identifying the true botanical disease in plants and plant leaves. In this way botany students can easily identify the plant leaf specie

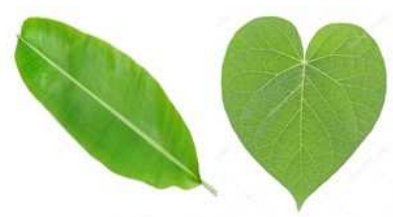

Banana leaf Bean leaf

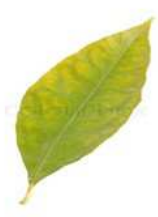

Lemon leaf

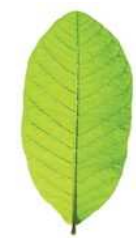

Guava leaf
Fig. 1. Types of Leaves

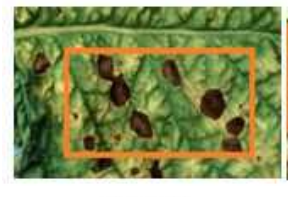

Alternata

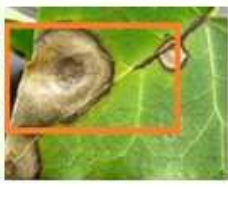

Anthracnose

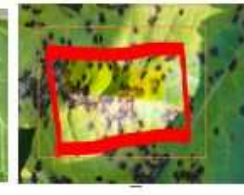

Bacterial Blight
Fig. 2. Types of Three Major Diseases in Leaves

and classify the type of disease in order to conclude the major causes for that particular damage and suggest a proper dosage administered to infected leaves. This paper provides an extension in agriculture research for identification of plant leaf and different disease of leaves.

Numerous literature review recommend that there are three main diseases that have resemblance with each other i.e., Alternata, Anthracnose and Bacterial blight. Therefore, this research emphases on diseases mentioned above with four different leaf species namely, bean leaf, lemon leaf, banana leaf and guava leaf as demonstrated in Fig. 1 and 2.

The paper presents to classify the leaf type as well as the disease through support vector machine (SVM). The algorithm proposed in this paper has been operated to identify 3 major diseases namely, alternata, anthracnose and bacterial blight with classification of selected leaf types such as leaf of beans, lemon, banana and guava as illustrated below:

From Fig. 1, one may find the type of leaf, whereas Fig. 2 presents three major diseases stated above. The first disease Alternata or sometimes known as Alternaria is a disease in which leaf may experience the black spots of major diameter. If the spots are smaller in size, it means that it is bacterial blight disease. The disease named Anthracnose is different from both diseases. In Anthracnose, the spots on the leaf are very big and within black spots, one may find another yellow-whitish spot as illustrated in Fig. 2. 


\section{LITERATURE REVIEW}

Leaves are the natural creation and may vary in terms of their physical appearances. To classify them is one of the biggest challenges and one cannot classify them without any botanist's suggestions. Botanist in terms of their shape, color, veins and skeleton have previously classified these leaves. In majority findings, one may get the shape-based recognition algorithms which gets huge failure if one has incomplete sample of particular leaf [1], [2]. Several techniques that have been proposed before may possess accuracy rate more than $65 \%$ [3]. The modern intelligent machine algorithms are also popular which have been proposed to sort out the fruits such as apple in terms of their color like red, normal red and poor red etc. [4]. Moreover, one finds the statistical and neural network-based classification for the detection of citrus diseases using machine vision. This particular algorithm had 90 percent accuracy [5] whereas moving classifier provides better results and have accuracy of $92 \%$ [6]. Studying the research papers of last few years, one may find curvelet transform technique that is used mostly for feature extraction [7]. If this data is analyzed properly and given to SVM based algorithm for training, then one can classify the type of leaf [8].

Curvelet transform has higher dimension of wavelet transform that has a provision to analyze the signal simultaneously in time as well as in spatial domain [9]. In majority of the papers, this transform is used to represent the images at different angle of orientations and scales. Since the advantage or the utility of using this transform is very simple, it is used for the discontinuous signals hence in our proposed case of leaves this will be a perfect fit [10]. In addition to this, curvelets remain coherent waveforms in a smooth medium and are based on band pass filtering in order to isolate the useless scales [11].

Various research manuscripts suggest the implementation of wavelets for the identification of pest damage on fruits in orchards [12]. Moreover, the morphological based features were also extracted in many research solutions in order to classify plants and early diagnosis of certain plant diseases [13]. Nowadays, when people are aware of neural networkbased classifiers, one may find artificial vision-oriented algorithms with fuzzy surface selection technique for disease prediction in plants [14]. While doing the brief literature review, one may come across several techniques for solving the disease and leaf type recognition such as conventional multiple regression and artificial neural networks (ANN).It is concluded in various papers that SVM based regression approach provides better description of disease whereas back propagation neural network provides the better results to identify the leaf in terms of its shape [15].

Edge detection-based image segmentation is also one of the most important techniques, which is frequently proposed for the cotton disease detection such algorithm has been quoted as homogeneous pixel counting technique for cotton disease detection [16]. In one of the proposed papers, K-mean based clustering is used to identify the defected areas, once the areas are identified then these defected features are extracted through color co-occurrence method and again used neural networks (NN) algorithm for identification and classification [17].

After studying image processing-based papers one can

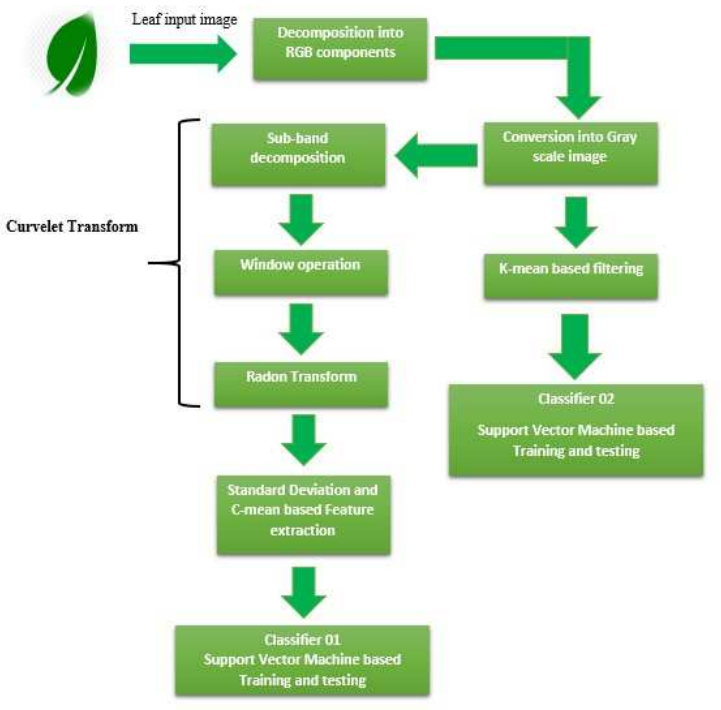

Fig. 3. Block Diagram of Proposed Algorithm

easily conclude that it has great significance and applications in the area of agricultural domain, one can also identify the grape fruit peel disease using the "SGDM" technique called as spatial gray level dependence matrices [18]. Moreover, Hue saturation (HS) along with fuzzy C-mean technique can also be used to extract the shade of leaf mask and shape [19]. This is illustrated by high-resolution multispectral technique, one can design automatic algorithm to categorize infected leaves, but could not possible to recognize leaf type species [20].

Moreover, the identification of leaf species and its disease can be known by computing the parameters such as inverse difference moment, correlation, entropy sum and variance [21]. In some of the proposed researches [22], "GLCM" abbreviate gray level co-occurrence matrix has been proposed to determine the parameters like energy and entropy of selected image. Whereas, tangential direction (TD) based segmentation is one of the proposed methods and in this method one can not only classify the healthy grape leaf from unhealthy one using K-Nearest Neighbor or K-Th Nearest Neighbor $(\mathrm{KNN})$ classification method but it also shares the direction and position of leaves. After studying several research manuscript, it is concluded that one can do same analysis using thresholding technique [23]. In this technique, one may compute the statistical values for the image of potato leaf and may concluded the healthiness of the leaf with $96 \%$ accuracy.

\section{Methodology}

The proposed algorithm in our work does not only provide the leaf species recognition but in addition to this, it also proposes a novel methodology to detect the 3 major diseases as well. The systematic designed method is provided in Fig. 3.

In the first step, the entire input digital image will be preprocessed in order to remove the noise and later on pixel enhancement will take place. Secondly, this RGB image will be changed into gray scale transformation so that the intensity levels can be reduced up to 255 levels. Once the image is converted into gray-scale then the curvelet transform will be 
applied. The applied curvelet transform is divided further into 4 sub-steps as follows.

\section{A. Decomposition into Sub-Bands}

In this sub step, the image matrix will be decomposed into various layers. In this way the whole frequency of the image will be divided into various sub-frequencies which can be later on added together to get the original image frequency as shown:

$$
f=f 1+f 2+f 3 \cdots
$$

Where frequency decomposition has been performed to compute the bands with specific frequencies ( $f 1, f 2, f 3)$. RGB image is sub-divided into layers in order to break them in to several frequencies, so that the sub-divided levels or layer with much noise and have spots in real can be filtered out.

\section{B. Dissection of Image}

Here the decomposed image is dissected further to run window operation for smooth partitioning,

$$
G q=w q \cdot f i
$$

Here $G q$ is a new matrix image that would store the window operated data after being multiplicative with smoothing frequency $f i$. The $w q$ is again a matrix of $6 \times 6$ that would run on whole image with $f i$ smoothing frequency in order to smooth the intensity levels.

\section{Normalization}

Normalization is the process that basically increases or adjust the contrast of image because of further decision. This is mainly performed to keep each dyadic square to the unit square.

\section{Ridgelet Analysis}

After normalizing, the image slices is used to catch monodimensional singularities, 1-D wavelet transform named as radon transform is used in bi-dimensional domain.

$$
\alpha(q, \lambda)=<g q, P \lambda>
$$

Where, $P, \lambda$ is an orthonormal set for $L^{2}\left(R^{2}\right)$ and $q$ is the collection of smooth windows $w q(x 1, x 2)$ localized around dyadic squares. After going through these sub steps our image is now enough ready to apply c-mean and standard deviation principle to extract the features. Furthermore, these features are then stored in a matrix i.e. leaf_specie.

The paper also computes 3 major diseases by implementing the K-mean based filtering to extract damage features and similarly storing it into another matrix named as disease_leaf. The algorithm stores these two types of features to run a moving classifier based on SVM to train and test the images for desired results.
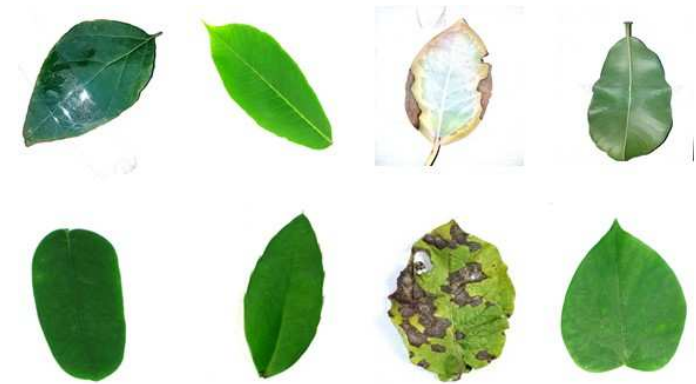

Fig. 4. Sample data set of leaves of mainly 3 leaf species

\begin{tabular}{|c|c|c|c|c|}
\hline \# & Feature & Classifier & Validation & Accuracy \\
\hline 1 & $\begin{array}{l}\text { Curvelet Transform } \\
{[8]}\end{array}$ & $\begin{array}{l}\text { Kernel Support Vec- } \\
\text { tor Machine (SVM) }\end{array}$ & $\begin{array}{l}\text { Can be processed } \\
\text { for individual leaf }\end{array}$ & $93.5 \%$ \\
\hline 2 & $\begin{array}{l}\text { Wavelet Transform } \\
\text { [24] }\end{array}$ & $\begin{array}{l}\text { Kernel Support Vec- } \\
\text { tor Machine (Radial } \\
\text { Basis Function) }\end{array}$ & Not validated & $93.07 \%$ \\
\hline \multirow[t]{2}{*}{3} & $\begin{array}{l}\text { Radial Basis Func- } \\
\text { tion [25] }\end{array}$ & $\begin{array}{l}\text { Back Proportional } \\
\text { Neural Network }\end{array}$ & $\begin{array}{l}\text { Can be processed } \\
\text { for individual leaf }\end{array}$ & $93.07 \%$ \\
\hline & $\begin{array}{l}\text { Radial Basis Func- } \\
\text { tion [25] }\end{array}$ & $\begin{array}{l}\text { Kernel Support Vec- } \\
\text { tor Machine (SVM) }\end{array}$ & $\begin{array}{l}\text { Kernel SVM (Ra- } \\
\text { dial Basis Func- } \\
\text { tion) }\end{array}$ & $97 \%$ \\
\hline
\end{tabular}

TABLE I. REPORTED Methods FOR LEAF SPECIE AND Disease RECOGNITION

\section{RESUlTS}

The algorithm will run twice in a go, and uses two classifiers based on SVM to compute the parameters like variance, difference in frames, entropy and energy of every level using histogram. These parameters are then fed to the two classifiers with two different nested if else conditions to compute first the leaf species, and once species is computed then second classifier will compute the disease.

The two classifiers based on SVM are prepared with the computation of 4 major parameters such as energy of the image, entropy, difference moment and variance of the image intensity levels. While training and testing this algorithm, 4 different plant leaf species and 3 major diseases were examined. The image pixels were varied but the resolution was same $640 \times 280$ as illustrated in Fig. 4 .

The training took place by taking total number of 640 leaf-based dataset. In this dataset, there had been 340 images that had been captured at run time. The computed results not only recognizes the leaf species but also recognize the disease infected by it through SVM learning algorithm based on mainly curvelet transform.

While computing the results, few important things were kept same such as the images were captured with digital camera by high resolution and with similar distance in a dark and white background. The comparative results of various species recognition with diseases have been illustrated in Table I. It presents the comparative analysis of various experiments done in this field of automated plant leaf species and disease recognition. It also focuses on different ways of feature extraction techniques used to extract the leaf features for classification in terms of accuracy.

In Fig. 5, it is clearly seen that an image of leaf has been captured is RGB in nature. Moreover this image is later 


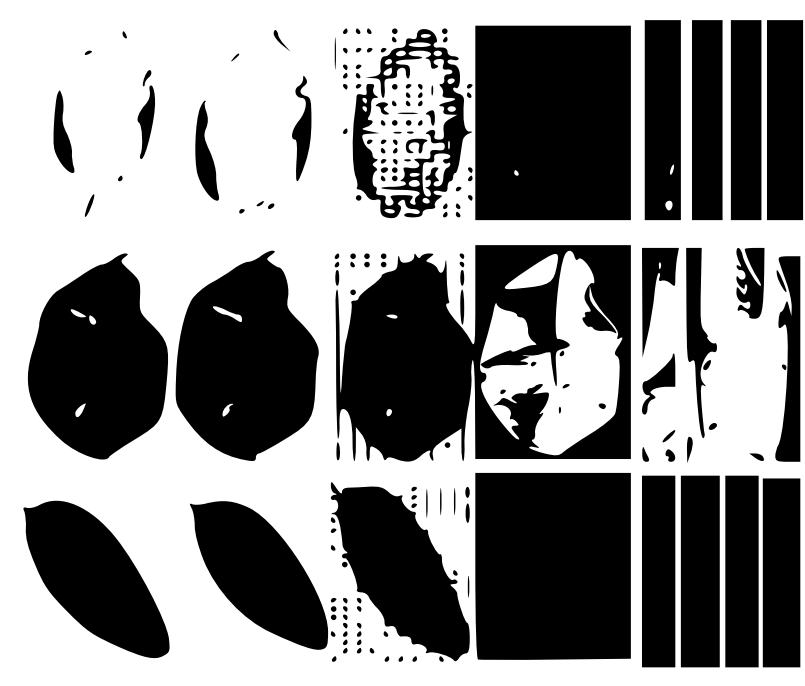

Fig. 5. Image Analysis using Proposed Algorithm

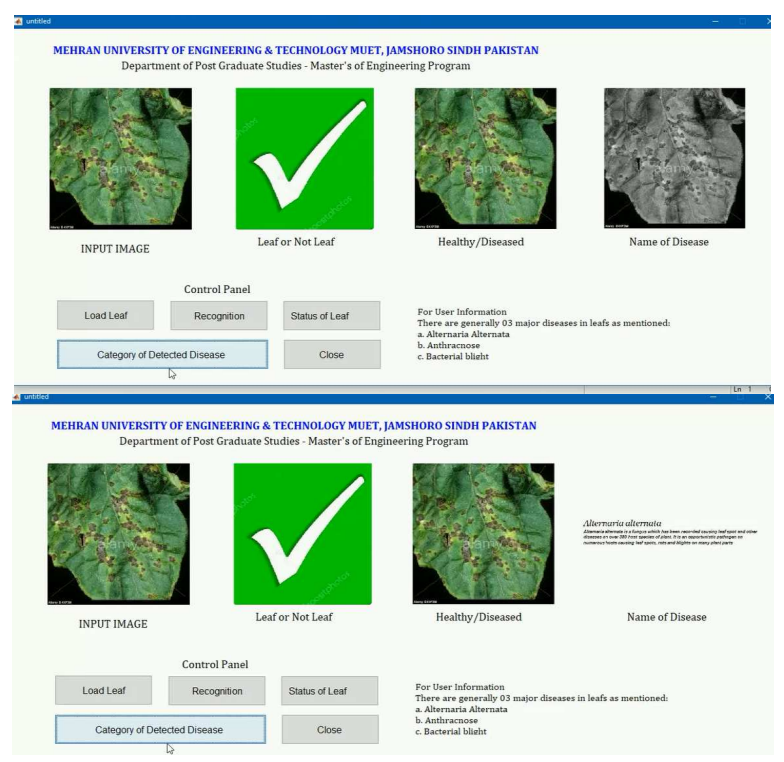

Fig. 6. GUI for Leaf Specie and disease recognition using Classifier

converted into gray scale and subdivided in to sub-layers. By doing this one can go for 3 sub-steps namely dissection, normalization, ridgelet analysis. The parameters as mentioned earlier are computed and on these parameters two classifiers are trained in order to identify the specie and disease.

In Fig. 6, the same process as shown in the block diagram of Fig. 3 is repeated on special graphical user interface and turned into MATLAB based application using MATLAB compiler mcc- command that basically invokes the compiler and provide a stand alone operation of the desired application.

\section{CONCLUSION}

The research work uses curvelet transform feature extraction and k-mean filtering based algorithm to detect leaf species and disease detection. The proposed research work is able to identify 3 major diseases successfully and its extensive analysis suggest that the algorithm is stable and provides better results comparatively on selected problem.
The presented approach focused three diseases for a specific plant leaves and in future more diseases could be added. The dataset of leaf images can be increased. Moreover, researcher may adopt another stated approach for disease detection.

\section{REFERENCES}

[1] J. Hossain and M. A. Amin. Leaf shape identification based plant biometrics. In 13th International Conference on Computer and Information Technology (ICCIT), pages 458-463, Dec 2010.

[2] Ji-Xiang Du, Xiao-Feng Wang, and Guo-Jun Zhang. Leaf shape based plant species recognition. Applied Mathematics and Computation, 185(2):883 - 893, 2007. Special Issue on Intelligent Computing Theory and Methodology.

[3] T. G. Crowe and M. J. Delwiche. Real-time defect detection in fruit - part ii: An algorithm and performance of a prototype system. Transactions of the ASAE, 39(6):2309-2317, 1996

[4] Kazuhiro Nakano. Application of neural networks to the color grading of apples. Computers and Electronics in Agriculture, 18(2):105 116, 1997. Applications of Artificial Neural Networks and Genetic Algorithms to Agricultural Systems.

[5] W.M Miller, J.A Throop, and B.L Upchurch. Pattern recognition models for spectral reflectance evaluation of apple blemishes. Postharvest Biology and Technology, 14(1):11 - 20, 1998.

[6] Jing Liu, Shanwen Zhang, and Jiandu Liu. A method of plant leaf recognition based on locally linear embedding and moving center hypersphere classifier. In De-Shuang Huang, Kang-Hyun Jo, Hong-Hee Lee, HeeJun Kang, and Vitoantonio Bevilacqua, editors, Emerging Intelligent Computing Technology and Applications. With Aspects of Artificial Intelligence, pages 645-651, Berlin, Heidelberg, 2009. Springer Berlin Heidelberg.

[7] J. Zhang, Z. Zhang, W. Huang, Y. Lu, and Y. Wang. Face recognition based on curvefaces. In Third International Conference on Natural Computation (ICNC 2007), volume 2, pages 627-631, Aug 2007.

[8] S. Prasad, P. Kumar, and R. C. Tripathi. Plant leaf species identification using curvelet transform. In 2nd International Conference on Computer and Communication Technology (ICCCT-2011), pages 646-652, Sep. 2011.

[9] V. Premalatha and S. R. Sannasi Chakravarthy. A low power bit-width adapted dct architecture for image processing applications. Digital Image Processing, 9(6):109-114, 2017.

[10] Gyanendra K. Verma, Shitala Prasad, and Gohel Bakul. Robust face recognition using curvelet transform. In Proceedings of the 2011 International Conference on Communication, Computing \&\#38; Security, ICCCS '11, pages 239-242, New York, NY, USA, 2011. ACM.

[11] Emmanuel J. Candes and David L. Donoho. Curvelets: A surprisingly effective nonadaptive representation for objects with edges. Technical report, Stanford University - Department of Statistics, Nov. 1999.

[12] Brendon J. Woodford, Nikola K. Kasabov, and C. Howard Wearing. Fruit image analysis using wavelets. In In Proceedings of the Iconip/Anziis/Annes, pages 88-91. University of Otago Press, 1999.

[13] Mohammad Ei-Helly, Ahmed Rafea, Salwa Ei-Gamal, and Reda Abd $\mathrm{Ei}$ Whab. Integrating diagnostic expert system with image processing via loosely coupled technique. Central Laboratory for Agricultural Expert System (CLAES), page 15, 2004.

[14] Rakesh Kaundal, Amar S. Kapoor, and Gajendra PS Raghava. Machine learning techniques in disease forecasting: a case study on rice blast prediction. BMC Bioinformatics, 7(1):485, Nov 2006.

[15] M. S. Prasad Babu and B.Srinivasa Rao. Leaves recognition using back propagation neural network-advice for pest and disease control on crops. IndiaKisan. Net: Expert Advisory System, 2007.

[16] Jayamala K. Patil and Raj Kumar. Advances in image processing for detection of plant diseases. Journal of Advanced Bioinformatics Applications and Research, 2(2):135-141, 2011. http://www.bipublication.com.

[17] H. Al-Hiary, S. Bani-Ahmad, M. Reyalat, M. Braik, and Z. ALRahamneh. Fast and accurate detection and classification of plant diseases. International Journal of Computer Applications, 17(1):31-38, March 2011. 
[18] Dae Gwan Kim, Thomas F. Burks, Jianwei Qin, and Duke M. Bulanon. Classification of grapefruit peel diseases using color texture feature analysis. International Journal of Agricultural and Biological Engineering (IJABE), 2(3):41-50, September 2009.

[19] Mohammed El-Helly, Ahmed A. Rafea, and Salwa El-Gammal. An integrated image processing system for leaf disease detection and diagnosis. In Bhanu Prasad, editor, Indian International Conference on Artificial Intelligence (IICAI), pages 1182-1195. IICAI, 2003.

[20] Sabine D. Bauer, Filip Korč, and Wolfgang Förstner. The potential of automatic methods of classification to identify leaf diseases from multispectral images. Precision Agriculture, 12(3):361-377, Jun 2011.

[21] T Vijayashree and A Gopal. Leaf identification for the extraction of medicinal qualities using image processing algorithm. In Intelligent Computing and Control (I2C2), 2017 International Conference on, pages 1-4. IEEE, 2017.

[22] N Krithika and A Grace Selvarani. An individual grape leaf disease identification using leaf skeletons and knn classification. In Innovations in Information, Embedded and Communication Systems (ICIIECS),
2017 International Conference on, pages 1-5. IEEE, 2017.

[23] Rudransh Sharma, Anushikha Singh, Malay Kishore Dutta, Kamil Riha, Petr Kriz, et al. Image processing based automated identification of late blight disease from leaf images of potato crops. In Telecommunications and Signal Processing (TSP), 2017 40th International Conference on, pages 758-762. IEEE, 2017.

[24] Jiandu Liu, Shanwen Zhang, and Shengli Deng. A method of plant classification based on wavelet transforms and support vector machines. In De-Shuang Huang, Kang-Hyun Jo, Hong-Hee Lee, Hee-Jun Kang, and Vitoantonio Bevilacqua, editors, Emerging Intelligent Computing Technology and Applications, pages 253-260, Berlin, Heidelberg, 2009. Springer Berlin Heidelberg.

[25] Shitala Prasad, Krishna Mohan Kudiri, and R. C. Tripathi. Relative sub-image based features for leaf recognition using support vector machine. In Proceedings of the 2011 International Conference on Communication, Computing \&\#38; Security, ICCCS '11, pages 343346, New York, NY, USA, 2011. ACM. 